\title{
Prediction of Area Under the Curve of Vancomycin in Infants: A Comparison of Two Methods
}

\author{
Nithya J Gogtay ${ }^{1} \cdot$ Sandeep B Bavdekar ${ }^{2}$ \\ Received: 23 February 2020 / Accepted: 6 March 2020 /Published online: 25 March 2020 \\ (C) Dr. K C Chaudhuri Foundation 2020
}

Therapeutic drug monitoring (TDM) involves measurement of drug concentrations in biologic fluids for improving patient outcomes through maximization of effectiveness and minimization of toxicity. The use of TDM with appropriate medical interpretation, directly impacts drug prescribing [1]. The fundamental premise of TDM is that the concentration (rather than the dose) is a better metric for the concentration at the site of action and thus a useful surrogate of both efficacy and toxicity [2]. A large body of evidence generated over the past four decades informs clinicians and policy makers about the concentration- effect relationship of antimicrobials [3]. However, the use of this metric is limited by lack of assay facilities, limited clinical outcome studies, missing population MIC (minimum inhibitory concentration) values and physician reluctance and their discomfort with modelling for prediction. In addition, just like dosing regimens in children are often derived from those in adults and do not necessarily reflect pediatric pharmacokinetics, TDM in children is an understudied area. The article published by Alsultan A and colleagues should thus be seen against this backdrop [4].

The authors found that the area-under-the curve [AUC, a measure of drug exposure] calculation for vancomycin using the two-sample [peak and trough] equation-based strategy to be a better predictor relative to the linear regression strategy that used trough levels alone in 70 infants [4]. It stands to reason that two variables are better than one giving the twosample strategy a prediction advantage. However, obtaining samples at two distinct time points in young infants may not be practical.

Sandeep B Bavdekar

sandeep.bavdekar@gmail.com

1 Department of Clinical Pharmacology, Seth GS Medical College and KEM Hospital, Mumbai, India

2 Department of Pediatrics, Surya Hospitals, Mumbai, India
Several aspects of methodology and analysis also remain unclear. The readers would have benefitted from information regarding the indication and clinical status of patients. The data appears to be a mix of patients from the intensive care unit (63\% of the study group) and other in-patients. It would have been better if a sub-group analysis was carried out to determine if the results are equally applicable to these distinct sub-populations. The choice of the paper by Le et al. for calculation of the reference AUC is fraught with an inherent challenge as Le et al. have studied older children with median age of $6.6 \mathrm{y}$ (IQR: 2.2 $13.4 \mathrm{y}$ and $78 \%$ of participant population over the age of two years) and a median body weight of $22.5 \mathrm{~kg}$ (IQR: $12.6-46 \mathrm{~kg}$ ) [5]. Also, while reporting AUCs, a distinction between reference and predicted AUCs has not been made at several places in the manuscript. A diagram depicting the relationship between reference AUC and predicted AUC using the linear regression method would have helped. Vancomycin concentrations in Table 1 in the article by Alsultan et al. [4] do not reflect the proportion of patients with sub-therapeutic, therapeutic and supra-therapeutic concentrations. This data would have helped understand Table 2 in that article [4] better, where the correlates of three concentration ranges with the proportion of patients with AUC $>400 \mu \mathrm{g} \mathrm{h}^{-1} \mathrm{ml}^{-1}$ has been made.

The findings reported by Alsultan and colleagues though remain relevant [4]. Vancomycin is the cornerstone antimicrobial therapy in the management of severe Gram-positive infections. Inappropriate vancomycin dosing is associated with therapeutic failure, bacterial resistance and toxicity. Thus, a study that goes beyond the measurement of drug concentrations into the realm of AUC calculation is appreciable. Such reportage, we hope, will kindle the interest of pediatricians and clinical pharmacologists in generating TDM data in children. This will hopefully, initiate the process whereby pediatricians, clinical pharmacologists, and microbiologists become more involved in supporting therapeutic decisions at least in special patient groups such as infants and children admitted in the ICUs. 


\section{Compliance with Ethical Standards}

Conflict of Interest None.

\section{References}

1. Touw DJ, Neef C, Thomson AH, Vinks AA. Cost-effectiveness of therapeutic drug monitoring: a systemic review. Ther Drug Monit. 2005;27:10-7.

2. Spector R, Park GD, Johnson GF, Vesell ES. Therapeutic drug monitoring. Clin Pharmacol Ther. 1988;43:345-53.
3. Reeves $\mathrm{D}$, Lovering $\mathrm{A}$, Thomson A. Therapeutic drug monitoring in the past 40 years of the journal of antimicrobial chemotherapy. J Antimicrob Chemo. 2016;71:3330-2.

4. Alsultan A, Abouelkheir M, Albassam A, Alharbi E, Assiri A, Alqahtani S. AUC- vs. trough-guided monitoring of vancomycin in infants. Indian J Pediatr. 2020. https://doi.org/10.1007/s12098-01903162-5.

5. Le J, Bradley JS, Murray W, et al. Improved vancomycin dosing in children using area-under-the-curve exposure. Pediatr Infect Dis J. 2013;32:e155-63.

Publisher's Note Springer Nature remains neutral with regard to jurisdictional claims in published maps and institutional affiliations. 